\title{
Analysis of non-obstetric vaginal and vulvar trauma: risk factors for operative intervention
}

\author{
Sahil Gambhir ${ }^{1}$ (D) Areg Grigorian $^{1} \cdot$ Sebastian Schubl ${ }^{1} \cdot$ Cristobal Barrios $^{1} \cdot$ Nicole Bernal $^{1} \cdot$ Victor Joe $^{1}$. \\ Viktor Gabriel ${ }^{1} \cdot$ Jeffry Nahmias ${ }^{1}$
}

Received: 5 June 2019 / Accepted: 5 September 2019 / Published online: 19 September 2019

(C) Italian Society of Surgery (SIC) 2019

\begin{abstract}
Vaginal and vulvar trauma may occur accidentally or because of an act of violence. Due to its rarity, little is known about risk factors effecting need for operative intervention. We sought to perform a large descriptive analysis of adult non-obstetric vulvovaginal trauma (VVT) and elucidate risk factors for requiring operative intervention. A retrospective analysis of the National Trauma Data Bank was performed between 2007 and 2015. Patients $\geq 16$ years old with vaginal or vulvar trauma were identified. Risk factors for surgical intervention were identified using a multivariable logistic regression analysis. From 2,040,235 female patients, $2445(<0.2 \%)$ were identified to have VVT with the majority being injury to the vagina $(68.6 \%)$. In patients with injury to the vagina, age $>65(\mathrm{OR}=0.41$, CI $0.26-0.62, p<0.001)$, Injury Severity Score $>25(\mathrm{OR}=0.66$, CI $0.50-0.86, p=0.01)$ and victims of rape ( $\mathrm{OR}=0.39,95 \% \mathrm{CI} 0.26-0.57, p<0.001)$ were less likely to require operative intervention. In patients with injury to the vulva, age $>65$ ( $\mathrm{OR}=0.45$, CI $0.21-0.94, p=0.02)$, victims of rape $(\mathrm{OR}=0.26$, CI $0.08-0.87, p=0.01)$ and gunshot violence $(\mathrm{OR}=0.10, \mathrm{CI} 0.02-0.59, p=0.02)$ were less likely to require operative intervention, but those with a concomitant injury to the vagina were more likely to require operative intervention $(\mathrm{OR}=2.56, \mathrm{CI}$ $1.63-4.03, p<0.001)$. Injuries to the vagina or vulva occur in $<0.2 \%$ of traumas. Interestingly, in both vulvar and vaginal trauma, older age, and involvement in rape were associated with lower risk for operative intervention. A combined injury to the vagina and vulva increases the need for operative intervention.
\end{abstract}

Keywords Vaginal $\cdot$ Vulvar $\cdot$ Trauma $\cdot$ Operative intervention

\section{Introduction}

Non-obstetric vulvovaginal trauma (VVT) occurs in less than $3.7 \%$ of trauma and the associated morbidity and mortality is rarely reported $[1,2]$. The reason for the rarity of this injury is related to the location within the perineum. However, the vulva and vagina are susceptible to hemorrhage due to their rich blood supply by branches of the internal pudendal artery [3]. The etiology of non-obstetric VVT includes sports-related injuries (bicycle related being the

Meeting presentation: Academic Surgical Congress, Houston, Texas 02/2019.

Sahil Gambhir

Sgambhi1@uci.edu

1 Department of Surgery, University of California Irvine Medical Center, 333 City Bldg. West, Suite 1600, Orange, CA 92868, USA most common), straddle injuries, midline splitting injuries (such as falls during in-line skating), crush injuries, penetrating injuries (including gunshot wounds), burns, ani$\mathrm{mal} /$ human bites, and non-accidental trauma such as sexual assault, rape, or abuse [4, 5].

There appears to be a paucity of data regarding nonobstetric VVT as many studies combine obstetric-related injuries with traumatic injuries [4, 6]. The majority of previous non-obstetric VVT studies focus on the pediatric population as the incidence is higher, which is attributed to the decreased labial fat pads that provide lesser protection [4]. Additionally, the majority of reports on adult VVT are small single-center studies, often reported as a case-series [7-10]. Furthermore, there appears to be minimal data within the current literature regarding risk factors for requiring operative intervention after VVT. To our knowledge, there are no management guidelines for non-obstetric VVT. Based on several case-series, it is postulated that risk factors for operative intervention after VVT are size of injury, rate of 
expansion (i.e., enlarging hematoma) and hypotension [3, $7,8,11]$.

Therefore, we sought to perform a large national descriptive analysis of adult VVT to determine possible risk factors for requiring operative intervention. We hypothesized hypotension as the largest risk factor associated with operative intervention for non-obstetric VVT.

\section{Methods}

This study was approved by the Institutional Review Board at the University of California, Irvine Medical Center. We performed a retrospective analysis of female trauma patients aged 16 or older admitted with vaginal or vulvar trauma using the National Trauma Data Bank (NTDB) from January 2007 to December 2015. All data from this study were obtained from NTDB and is available through their permission. Vaginal trauma was defined using the International Classification of Diseases (ICD) Version-9 diagnosis codes: 878.4, 878.5. Vulvar trauma was defined using the ICD-9 diagnosis codes: 878.6, 878.67.

Our primary end-point was operative intervention, defined as patients undergoing surgery due to injury. Vaginal operative intervention was defined using the ICD-9 procedure codes as 70.62-70.91, 70.33, 70.4. Vulvar operative intervention was defined using the ICD-9 procedure codes as 71.4-71.8. Demographic characteristics, mechanism of injury, and injury-associated characteristics including Injury Severity Score (ISS) were collected. Demographics included age. Mechanisms of injury included rape, abuse, assault, and penetrating injury. The latter group was further subclassified by gunshot wound, stab wound or other. In addition to ISS, injury profile characteristics such as hypotension on arrival (systolic blood pressure $<90 \mathrm{mmHg}$ ) and severe abbreviated injury scale (AIS $>3$ ) were collected. Clinical outcomes measured included hospital length of stay (LOS), ventilator days, urinary tract infection and mortality.

Descriptive statistics were performed for all variables. A Student's $t$ test and Mann-Whitney $U$ test were used to compare continuous variables and Chi-square was used to compare categorical variables for bivariate analysis. Categorical data were reported as percentages, and continuous data were reported as medians with interquartile range (IQR) or as means with standard deviation (SD).

The magnitude of the association between risk factor variables and surgical intervention were first measured using a univariable logistic regression model. Covariates with statistical significance $(p<0.20)$ were included in a hierarchical multivariable logistic regression model and the adjusted risk for surgical intervention was reported with an odds ratio (OR) and $95 \%$ confidence intervals (CI). The reference group used in our analysis included patients who sustained vaginal and vulvar trauma, respectively, and did not undergo intervention. The magnitude of the association between risk factor variables and mortality were measured using a multivariable logistic regression model and the adjusted risk for mortality was reported with an OR and 95\% CI. The reference group used in our analysis included patients who sustained vaginal and vulvar trauma, respectively, and did not undergo intervention. Covariates for the patients with injury to the vagina were age $\geq 65$, hypotension on arrival, concomitant injury to the vulva, mechanism of injury, ISS $\geq 25$, and severe AIS grades to the head, thorax and abdomen, respectively. Covariates for the patients with injury to the vulva were age $\geq 65$, hypotension on arrival, concomitant injury to the vagina, mechanism of injury, ISS $\geq 25$, and severe AIS grades to the head, thorax and abdomen, respectively $[3,7,8,11]$. All $p$ values were two-sided, with a statistical significance level of $<0.05$. All missing data points were not imputed, but treated as missing data. All analyses were performed with IBM SPSS Statistics for Windows (Version 24, IBM Corp., Armonk, NY, USA).

\section{Results}

\section{Patient demographics}

From 2,040,235 female patients, $2445(<0.2 \%)$ were identified to have VVT. Of these, 1677 (68.6\%) had a vaginal injury, whereas 768 (32.4\%) had vulvar injury. Of the 1677 patients who had vaginal injury, 820 (48.9\%) did not undergo surgery, whereas 857 (51.1\%) underwent surgical intervention. Patients managed non-operatively with vaginal injury were older (median age 32 vs. 29 years old, $p=0.01$ ) and had a greater ISS (13.5 vs. 9.0, $p=0.01$ ) compared to those who underwent vaginal surgery. Those who underwent vaginal surgery and those managed non-operatively had similar rates of rape $(8.2 \%$ vs $10.7 \%, p=0.43)$. Of the 768 patients who had a vulvar injury, $486(63.3 \%)$ were managed non-operatively, whereas 282 (34.7\%) underwent surgical intervention. Patients managed non-operatively with a vulvar injury were older ( $31 \mathrm{vs.} 26$ years old, $p=0.02$ ), but had no difference in ISS (10.0 vs. 10.0, $p=0.24$ ) compared to those who underwent vulvar surgery. Patients who sustained vulvar injury and did not undergo surgery had higher rates of rape than those who underwent surgery $(5.1 \%$ vs. $1.4 \%, p=0.01)$. Irrespective of surgery, the most common concomitant injury to the vagina or vulva was pelvic fracture (Table 1). In total, 100 patients had combined vaginal and vulvar trauma. Of these 100 patients, $35(35.0 \%)$ were managed non-operatively, whereas $65(65.0 \%)$ underwent surgical intervention. 
Table 1 Patient characteristics of adult external genitalia trauma

\begin{tabular}{|c|c|c|c|c|}
\hline \multirow[t]{2}{*}{ Characteristic } & \multicolumn{2}{|c|}{ Injury to vagina $(n=1677)$} & \multicolumn{2}{|c|}{ Injury to vulva $(n=768)$} \\
\hline & - Surgery $(n=820)$ & + Surgery $(n=857)$ & - Surgery $(n=486)$ & + Surgery $(n=282)$ \\
\hline Age, y, median (IQR) & $32.0(26)$ & $29.0(23)^{*}$ & $31.0(24)$ & $26.0(19)^{*}$ \\
\hline ISS median (IQR) & $13.5(25)$ & $9.0(20)^{*}$ & $10.0(19)$ & $10.0(15)$ \\
\hline Hypotension on arrival $(\mathrm{SBP}<90)$ & 95 (11.6) & $85(9.9)$ & 37 (7.6) & $11(3.9)^{*}$ \\
\hline \multicolumn{5}{|l|}{ Mechanism, $n(\%)$} \\
\hline Rape & $88(10.7)$ & $70(8.2)$ & $25(5.1)$ & $4(1.4)^{*}$ \\
\hline \multicolumn{5}{|l|}{ Penetrating } \\
\hline Gunshot wound & $47(5.7)$ & $83(9.7) *$ & $60(12.3)$ & $18(6.4)^{*}$ \\
\hline Stab wound & $38(4.6)$ & $30(3.5)$ & $20(4.1)$ & $12(4.3)$ \\
\hline \multicolumn{5}{|l|}{ Blunt } \\
\hline Motor vehicle accident & $192(23.4)$ & $150(17.5)^{*}$ & $115(23.7)$ & $58(20.6)$ \\
\hline Bicycle accident & $21(2.6)$ & $5(0.6)^{*}$ & $25(5.1)$ & $12(4.3)$ \\
\hline Motorcycle accident & $125(5.3)$ & $58(6.5)$ & $44(9.1)$ & $25(8.9)$ \\
\hline \multicolumn{5}{|l|}{ Concomitant injuries, $n(\%)$} \\
\hline Bladder & $65(7.9 \%)$ & $(7.1 \%)$ & $34(7.0 \%)$ & $33(11.7 \%)^{*}$ \\
\hline Kidney & $31(3.8 \%)$ & $25(2.9 \%)$ & $16(3.3 \%)$ & $7(2.5 \%)$ \\
\hline Anorectal & $121(14.8 \%)$ & $125(14.6 \%)$ & $30(6.2 \%)$ & $32(11.3 \%)^{*}$ \\
\hline Pelvis fracture & $365(44.5 \%)$ & $306(35.7 \%)$ & $177(36.4 \%)$ & $111(39.4 \%)^{*}$ \\
\hline \multicolumn{5}{|l|}{ Severe $^{\mathrm{a}}$ AIS, $n(\%)$} \\
\hline Head & $80(9.8)$ & $40(4.7)^{*}$ & $45(9.3)$ & $7(2.5)^{*}$ \\
\hline Spine & $5(0.6)$ & $1(0.1)$ & $3(0.6)$ & $2(0.7)$ \\
\hline Thorax & 65 (7.9) & $35(4.1)^{*}$ & $19(3.9)$ & $8(2.8)$ \\
\hline Abdomen & $58(7.1)$ & $50(5.8)$ & $13(2.7)$ & $5(1.8)$ \\
\hline
\end{tabular}

$y$ years, ISS Injury Severity Score, IQR interquartile range, AIS Abbreviated Injury Score

*Statistically significant

${ }^{\text {a }}$ Severe (grade $\left.>3\right)$

\section{Multivariable analysis for risk of operative intervention in patients with injury to the vagina or vulva}

In patients with injury to the vagina, age $\geq 65(\mathrm{OR}=0.41$, CI $0.26-0.62, p<0.001)$, ISS $\geq 25$ (OR $=0.66$, CI $0.50-0.86$, $p=0.01)$ and victims of rape $(\mathrm{OR}=0.39,95 \%$ CI $0.26-0.57$, $p<0.001)$ were less likely to require operative intervention (Table 2). In patients with injury to the vulva, age $\geq 65$ $(\mathrm{OR}=0.45$, CI $0.21-0.94, p=0.02)$, victims of rape $(\mathrm{OR}=0.26$, CI $0.08-0.87, p=0.01)$ and gunshot violence $(\mathrm{OR}=0.10$, CI $0.02-0.59, p=0.02)$ were less likely to require operative intervention. However, those with a vulva injury and concomitant injury to the vagina were more likely to require operative intervention ( $\mathrm{OR}=2.56$, CI 1.63-4.03, $p<0.001$ ) (Table 3).

\section{Multivariable analysis for risk of mortality in patients with injury to the vagina or vulva}

In patients with injury to the vagina, those who underwent operative intervention had a significantly decreased risk of
Table 2 Multivariable analysis for risk of intervention in patients with injury to the vagina

\begin{tabular}{lllc}
\hline Risk factor & OR & CI & $p$ value \\
\hline Age $\geq 65$ & $\mathbf{0 . 4 1}$ & $\mathbf{0 . 2 6 - 0 . 6 2}$ & $<\mathbf{0 . 0 0 1}$ \\
Hypotension on arrival & 1.01 & $0.73-1.41$ & 0.94 \\
Concomitant injury to vulva & 1.19 & $0.78-1.82$ & 0.41 \\
Rape & $\mathbf{0 . 3 9}$ & $\mathbf{0 . 2 6 - 0 . 5 7}$ & $<\mathbf{0 . 0 0 1}$ \\
Abuse & 0.68 & $0.30-1.54$ & 0.35 \\
Other assault & 0.66 & $0.42-1.04$ & 0.07 \\
Motor vehicle accident & 1.03 & $0.77-1.39$ & 0.83 \\
Motorcycle accident & 1.21 & $0.79-1.85$ & 0.38 \\
Bicycle accident & $\mathbf{0 . 2 8}$ & $\mathbf{0 . 1 0 - 0 . 7 5}$ & $\mathbf{0 . 0 1}$ \\
Other blunt & $\mathbf{0 . 4 0}$ & $\mathbf{0 . 2 9 - 0 . 5 4}$ & $<\mathbf{0 . 0 0 1}$ \\
Gunshot wound & 1.63 & $0.70-3.78$ & 0.25 \\
Stab wound & 0.64 & $0.28-1.47$ & 0.29 \\
Other penetrating & 0.68 & $0.34-1.35$ & 0.27 \\
ISS $\geq 25$ & $\mathbf{0 . 6 6}$ & $\mathbf{0 . 5 0 - 0 . 8 6}$ & $\mathbf{0 . 0 1}$ \\
Severe abdominal AIS & 1.13 & $0.74-1.73$ & 0.58 \\
\hline
\end{tabular}

ISS Injury Severity Score, AIS Abbreviated Injury Score 
Table 3 Multivariable analysis for risk of intervention in patients with injury to the vulva

\begin{tabular}{lllc}
\hline Risk factor & OR & CI & $p$ value \\
\hline Age $\geq 65$ & $\mathbf{0 . 4 5}$ & $\mathbf{0 . 2 1 - 0 . 9 4}$ & $\mathbf{0 . 0 2}$ \\
Hypotension on arrival & 0.56 & $0.27-1.16$ & 0.12 \\
Concomitant injury to vagina & $\mathbf{2 . 5 6}$ & $\mathbf{1 . 6 3}-\mathbf{4 . 0 3}$ & $<\mathbf{0 . 0 0 1}$ \\
Rape & $\mathbf{0 . 2 6}$ & $\mathbf{0 . 0 8}-\mathbf{0 . 8 7}$ & $\mathbf{0 . 0 1}$ \\
Abuse & 0.26 & $0.05-1.30$ & 0.10 \\
Other assault & 1.02 & $0.47-2.21$ & 0.97 \\
Motor vehicle accident & 0.69 & $0.46-1.03$ & 0.07 \\
Motorcycle accident & 0.74 & $0.43-1.30$ & 0.30 \\
Bicycle accident & 0.61 & $0.29-1.26$ & 0.18 \\
Other blunt & 1.38 & $0.69-2.79$ & 0.37 \\
Gunshot wound & $\mathbf{0 . 1 0}$ & $\mathbf{0 . 0 2 - 0 . 5 9}$ & $\mathbf{0 . 0 2}$ \\
Stab wound & 0.20 & $0.03-1.24$ & 0.08 \\
Other penetrating & 5.26 & $0.93-29.75$ & 0.06 \\
ISS $\geq 25$ & 0.77 & $0.50-1.19$ & 0.24 \\
Severe abdominal AIS & 0.74 & $0.24-2.26$ & 0.59 \\
\hline
\end{tabular}

ISS Injury Severity Score, AIS Abbreviated Injury Score

mortality $(\mathrm{OR}=0.25$, CI $0.14-0.44, p<0.001)$ compared to those who underwent non-operative intervention.

In patients with injury to the vulva, those who underwent operative intervention had a significantly decreased risk of mortality $(\mathrm{OR}=0.07$, CI $0.01-0.63, p=0.018)$ compared to those who underwent non-operative intervention.

\section{Outcomes of patients with VVT}

Patients with vaginal trauma who underwent surgery had similar LOS (5.0 days vs. 5.0 days, $p=0.48$ ) and rate of UTI ( $2.6 \%$ vs. $2.3 \%, p=0.44$ ), compared to those who underwent non-operative management. Patients with vaginal trauma who underwent surgery had a decreased mortality rate relative to the non-operative cohort ( $2.1 \%$ vs. $10.7 \%, p<0.001$ ). Compared to patients with vulvar trauma that underwent surgery, patients who did not undergo surgery had a shorter LOS (5.0 vs. $4.0, p=0.01$ ). Patients with vulvar trauma who underwent surgery had a decreased mortality rate relative to the non-operative management group $(0.4 \%$ vs. $5.4 \%$, $p=0.01)$ (Table 4).

\section{Discussion}

Although non-obstetric VVT is rare, we present the largest descriptive analysis to date. Over 9 years, the incidence of non-obstetric VVT was $<0.2 \%$ and of these, over two-thirds were vaginal injuries, whereas less than one-third had vulvar injury. Interestingly, the mortality rate for both vaginal and vulvar trauma patients that underwent surgery was lower compared to those who were managed non-operatively. In addition, we demonstrated patients who sustained either vaginal or vulvar trauma, that were equal or older than 65 years and were victims of rape were associated with a decreased risk of operative intervention. However, the largest risk factor for operative intervention for patients with vulvar trauma was concomitant vaginal injury. With the exception of mortality, outcomes were relatively similar between operative and non-operative management after vaginal or vulvar trauma.

Non-obstetric VVT is an extremely rare occurrence, however the incidence is likely underreported. Our analysis revealed the incidence of VVT was $<0.2 \%$ with the majority occurring due to vaginal trauma and an overall mortality rate of over 5\%. Interestingly, the rates of mortality were decreased in both surgical cohorts after vaginal and vulvar trauma compared to non-operative cohorts by $8.6 \%$ and $5.0 \%$, respectively. The reason for this increased rate of mortality in the non-operative group is likely selection bias as the non-operative group had a higher AIS head in both groups, which may have skewed the results. In fact, after controlling for AIS head among other variables, we found the risk of mortality was decreased in patients with VVT who underwent operative intervention relative to those who underwent non-operative intervention. Moreover, our reported incidence should be viewed with caution as our analysis is based on trauma patients that are incorporated in

Table 4 Patient outcomes of adult external genitalia trauma

\begin{tabular}{|c|c|c|c|c|c|c|}
\hline \multirow[t]{2}{*}{ Characteristic } & \multicolumn{3}{|l|}{ Injury to vagina } & \multicolumn{3}{|l|}{ Injury to vulva } \\
\hline & - Surgery $(n=820)$ & + Surgery $(n=857)$ & $p$ value & - Surgery $(n=486)$ & + Surgery $(n=282)$ & $p$ value \\
\hline \multicolumn{7}{|l|}{ Outcomes } \\
\hline LOS, days, median (IQR) & $5.0(12)$ & $5.0(9)$ & 0.48 & $4.0(8)$ & $5.0(9)$ & 0.01 \\
\hline ICU, days, median (IQR) & $5.0(10)$ & $4.0(7)$ & 0.43 & $4.0(7)$ & $5.0(6)$ & 0.40 \\
\hline Ventilator, days, median (IQR)) & $5.0(9)$ & $4.0(7)$ & 0.67 & $4.0(8)$ & $3.0(5)$ & 0.62 \\
\hline Urinary tract infection, $n(\%)$ & $19(2.3)$ & $22(2.6)$ & 0.44 & $13(2.7)$ & $12(4.3)$ & 0.58 \\
\hline Mortality, $n(\%)$ & $83(10.7)$ & $18(2.1)$ & $<0.001$ & $25(5.4)$ & $1(0.4)$ & 0.01 \\
\hline
\end{tabular}

$L O S$ length of stay, $I C U$ intensive care unit, $I Q R$ interquartile range 
the NTDB and therefore do not represent patients with isolated non-obstetric VVT such as patients who were victims of rape. This is supported by the fact our patient population comprised $25.4 \%$ of patients suffering from rape, whereas a single-center study by Jones et al. on the incidence of VVT found rape accounted for $70 \%$ of their population and had no deaths [1]. In addition, our finding that the most common concomitant injury for both VVT groups was a pelvic fracture provides important information for the bedside clinician to consider when they encounter VVT on physical examination.

The risk factors for operative intervention after nonobstetric VVT have not been previously elucidated. Previous case-series have considered size of injury, rate of expansion (i.e., enlarging hematoma) or hypotension due to hemorrhage as indicators for operative intervention after both obstetric and non-obstetric VVT [3, 7, 8, 11]. Contrary to these reports and our hypothesis, we found hypotension on arrival was not a risk factor after controlling for significant covariates. However, we found patients with concomitant injury to the vulva and vagina had greater than twofold increased risk of operative intervention. Similarly, in a retrospective study of pediatric patients who sustained accidental genital trauma (excluding sexual related injuries), Iqbal and colleagues reported the operative cohort were 35\% more likely to have multiple genital injuries [12]. Interestingly, we found in patients with injury to either vagina or vulva, age $\geq 65$ and victims of rape were less likely to undergo operative intervention. With regard to age, we can only postulate one of the reasons may be due to decreased desire for surgical intervention in elderly females. While not entirely similar, an epidemiology-based study from Australia analyzed patients who underwent vulvoplasty from 2001 to 2013 and found patients in the oldest age group had the least desire for intervention [13]. We also found patients with injury to the vagina and an ISS $\geq 25$ were associated with a decreased risk of operative intervention which we can only speculate represents a potential selection bias as these patients may not have been offered surgery due to the severity of trauma and other concomitant injuries. Future prospective research regarding VVT and consideration of size of injury and rate of expansion of injury is required to assist clinicians in management of VVT. To our knowledge, there are no management guidelines after non-obstetric VVT from large trauma based or obstetrics and gynecology associations/societies. Therefore, we call for action to perform future research to confirm our findings and elucidate other risk factors in order to develop guidelines for the management of non-obstetric VVT.

Outcomes of patients sustaining VVT may rely on management including non-operative and operative interventions [11]. Our analysis found nearly half of the patients with vaginal trauma and nearly two-thirds of the patients with vulvar trauma did not undergo operative intervention. Advocates for non-operative management especially when the VVT results in hematoma include the tamponading effects of the space limited anatomical areas. Interestingly, no differences were found when comparing the operative and non-operative cohorts for vaginal or vulvar injury with respect to ICU days, ventilator days or rate of UTI. The only difference found was a 1 day decrease in LOS for non-operative management after vulvar trauma. Whether this was directly due to the operative intervention or was a manifest of other factors such as concomitant injuries and increased mortality in the non-operative management group is unknown. Future studies are necessary to evaluate these findings.

Our study has several limitations including those inherent to a retrospective database study such as selection bias and miscoding. Furthermore, we are missing pertinent data points such as severity of vaginal or vulvar injury (i.e., American Association for the Surgery of Trauma injury grade), type of vaginal or vulvar injury (hematoma vs. laceration) and aspects of surgical intervention (i.e., exam under anesthesia, hemorrhage control, etc.). Also, our study is limited by details regarding the anatomical location of the VVT (e.g., anterior/posterior). In addition, injury data were abstracted from ICD 9 codes and therefore, we are not able to distinguish between hematoma or laceration secondary to VVT which may significantly influence need for intervention. The NTDB has several biases which include voluntary data submission which may omit key data, inherent quality improvement processes at the hospitals involved in the NTDB and finally, the availability of sub-specialists are not unified among all institutions participating in the NTDB. Another limitation of the NTDB is information regarding the specialty of the operating surgeon performing interventions (i.e., trauma surgeon vs. gynecologic surgeon) is unavailable. Finally, this database is limited to the index hospitalization and does not allow for any follow-up data including long-term morbidity/mortality and quality of life or cosmetic/functional outcomes.

\section{Conclusion}

This study is the largest analysis of non-obstetric VVT. VVT remains rare with a higher incidence of vaginal trauma relative to vulvar trauma. This study found older age and victims of rape were the most significant independent predictors of undergoing non-operative management for both vaginal and vulvar trauma. However, concomitant injury of the vagina and vulva was the largest independent risk factor for operative intervention for patients who sustained vulvar trauma. In addition, foregoing operative intervention is associated with higher rates of mortality after either vaginal or vulvar trauma, although this may reflect selection bias. Future 
prospective research is needed to validate the risk factors for operative intervention of the vagina and vulva found in this study, as well as identify other risk factors with the hopes of developing consensus guidelines that can be utilized by practicing clinicians.

\section{Compliance with ethical standards}

Conflict of interest The authors report no proprietary or commercial interest in any product mentioned or concept discussed in this article.

Ethical approval This article does not contain any studies with human participants or animals performed by any of the authors.

Informed consent For this type of study, informed consent is not required.

\section{References}

1. Jones IS, O'Connor A (2013) Non-obstetric vulval trauma. Emerg Med Australas EMA 25(1):36-39

2. Saxena AK, Steiner M, Hollwarth ME (2014) Straddle injuries in female children and adolescents: 10-year accident and management analysis. Indian J Pediatr 81(8):766-769

3. Ernest A, Knapp G (2015) Severe traumatic vulva hematoma in teenage girl. Clin Case Rep 3(12):975-978

4. Lopez HN, Focseneanu MA, Merritt DF (2018) Genital injuries acute evaluation and management. Best Pract Res Clin Obstet Gynaecol 48:28-39
5. Merritt DF (2004) Vulvar and genital trauma in pediatric and adolescent gynecology. Curr Opin Obstet Gynecol 16(5):371-381

6. Oliveira LS, Brito LG, Quintana SM, Duarte G, Marcolin AC (2014) Perineal trauma after vaginal delivery in healthy pregnant women. Sao Paulo Med J 132(4):231-238

7. Benrubi G, Neuman C, Nuss RC, Thompson RJ (1987) Vulvar and vaginal hematomas: a retrospective study of conservative versus operative management. South Med J 80(8):991-994

8. Propst AM, Thorp JM Jr (1998) Traumatic vulvar hematomas: conservative versus surgical management. South Med J 91(2):144-146

9. Sau AK, Dhar KK, Dhall GI (1993) Nonobstetric lower genital tract trauma. Aust N Zeal J Obstet Gynaecol 33(4):433-435

10. Fallat ME, Weaver JM, Hertweck SP, Miller FB (1998) Late follow-up and functional outcome after traumatic reproductive tract injuries in women. Am Surg 64(9):858-861

11. Gianini GD, Method MW, Christman JE (1991) Traumatic vulvar hematomas. Assessing and treating nonobstetric patients. Postgrad Med 89(4):115-118

12. Iqbal CW, Jrebi NY, Zielinski MD, Benavente-Chenhalls LA, Cullinane DC, Zietlow SP et al (2010) Patterns of accidental genital trauma in young girls and indications for operative management. J Pediatr Surg 45(5):930-933

13. Ampt AJ, Roach V, Roberts CL (2016) Vulvoplasty in New South Wales, 2001-2013: a population-based record linkage study. Med J Aust 205(8):365-369

Publisher's Note Springer Nature remains neutral with regard to jurisdictional claims in published maps and institutional affiliations. 